\title{
Serum levels of IL-1ra and sTNF RI in patients with uterine sarcoma
}

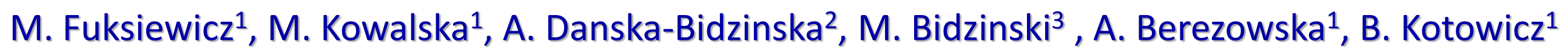

\footnotetext{
${ }^{1}$ Laboratory of Tumor Markers, Department of Pathology and Laboratory Diagnostics, Maria Sklodowska-Curie Memorial Cancer Centre and Institute of Oncology, Warsaw, Poland

2 Department of Obstetrics and Gynecology, Medical University of Warsaw, Warsaw, Poland.

${ }^{3}$ Department of Gynecological Oncology, The Maria Sklodowska-Curie Institute-Oncology Center in Warsaw, Poland
}

Introduction and Objectives: Tumor development and progression is often accompanied by overexpression of cytokines and their receptors in tumor and/or stromal cells, as well as in immune cells. Fundamental importance was a discovery that some cytokine receptors found on malignant cells determine their metastatic potential. Proinflammatory cytokines and their receptors play a crucial role in inflammation and their release may relate to the frequency of local recurrence of tumor. The purpose of this study was to evaluate the potential use of antagonist receptor of interleukin 1(IL-1ra) and soluble receptor TNF (sTNF RI) serum levels as a diagnostic marker in patients with uterine sarcoma.

PATIENTS AND METHODS: The study included 35 untreated uterine sarcoma patients, aged between 23-80 years (median 56 yours old), and 30 healthy women as controls. Concentrations of serum IL-1ra and STNF RI were determined by the ELISA of R\&D. Standard concentration for cytokines was determined taking the 95 percentile. For the statistical analysis, Mann-Whitney $U$ tests were applied. Receiver-operating characteristic (ROC) curves were used, to assess the diagnostic sensitivity and specificity of the marker.

Pretreatment serum sTNF RI II-1 ra and levels according to controls
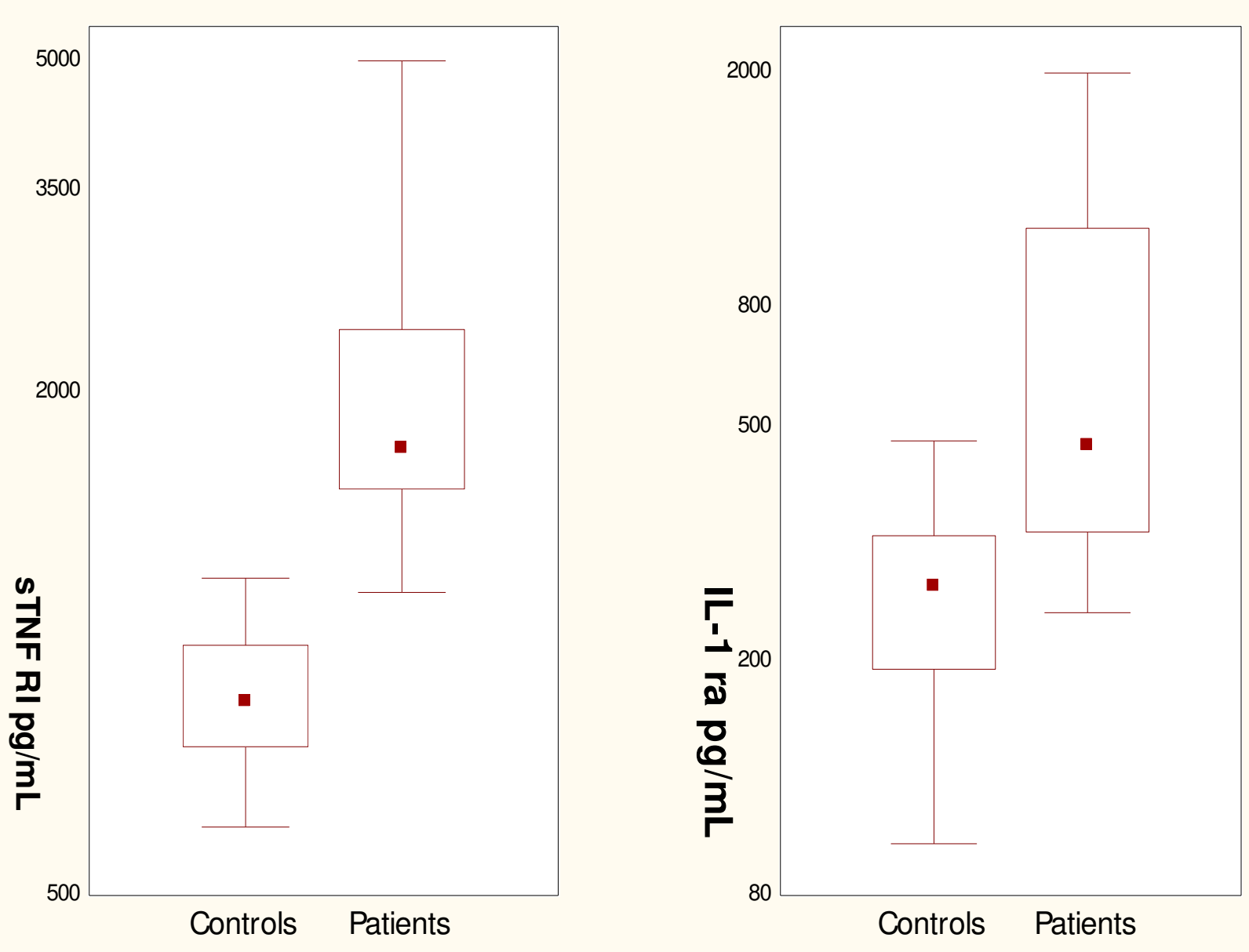

\section{RESULTS:}

$>$ We show a significant increase in both sTNF RI $(p<0,0001)$ and IL-1ra $(p<0,0001)$ in blood serum samples from patients with uterine sarcomas, as compared to control group of healthy female individuals.

Increased concentration of STNF RI was observed more frequently among patients (94\% of all patients) than IL-1ra (50\% of patients).

Based on ROC curve analysis, we observed higher accuracy of sTNF RI (AUC-0.998) than IL1ra (AUC=0.795) to detect uterine sarcoma.

\section{$>$ CONCLUSION: These results suggest} that determination of IL-1ra and sTNF RI serum levels may be a useful marker in patients with uterine sarcoma.

ROC curves of STNF RI and II-1ra in uterine sarcoma patients. Pairwise nonparametric comparisons of the neighboring AUCs were performed by the Wilcoxon test

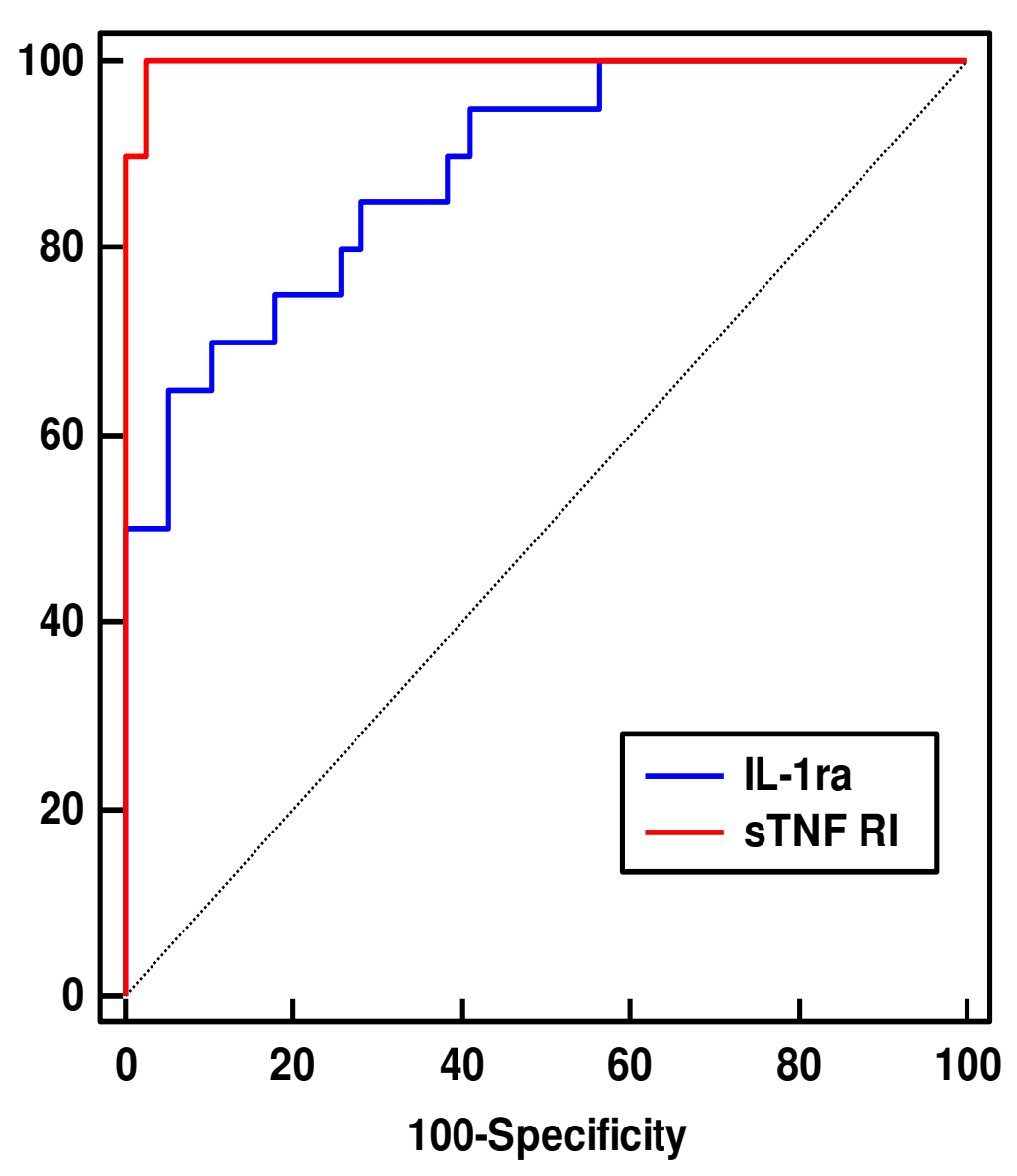

